\section{ISG15 modification of the eIF4E cognate 4EHP enhances cap structure-binding activity of 4EHP}

\author{
Fumihiko Okumura, Weiguo Zou, \\ and Dong-Er Zhang ${ }^{1}$
}

Department of Molecular and Experimental Medicine, The Scripps Research Institute, La Jolla, California 92037, USA

\begin{abstract}
The expression of the ubiquitin-like molecule ISG15 and protein modification by ISG15 (ISGylation) are strongly activated by interferon, genotoxic stress, and pathogen infection, suggesting that ISG15 plays an important role in innate immune responses. $4 \mathrm{EHP}$ is an mRNA 5' cap structure-binding protein and acts as a translation suppressor by competing with eIF4E for binding to the cap structure. Here, we report that 4EHP is modified by ISG15 and ISGylated 4EHP has a much higher cap structure-binding activity. These data suggest that ISGylation of 4EHP may play an important role in cap structuredependent translation control in immune responses.
\end{abstract}

Supplemental material is available at http://www.genesdev.org.

Received July 19, 2006; revised version accepted December 19, 2006.

ISG15 was the first reported ubiquitin-like modifier (ubl) (Haas et al. 1987). It forms covalent conjugates with cellular proteins similar to protein ubiquitylation (Loeb and Haas 1992). ISG15 expression and protein modification by ISG15 (ISGylation) are strongly activated by Type I interferon (IFN) (Farrell et al. 1979; Loeb and Haas 1992). IFNs are critical cytokines involved in innate immune responses (Garcia-Sastre and Biron 2006). These facts suggest that ISG15 modification may modulate certain immune responses related to pathogen infections and various stresses. Similar to protein ubiquitylation, ISGylation is also regulated by a set of enzymes. UBE1L is an ISG15 E1 enzyme, which shows high homology with the ubiquitin E1 (Yuan and Krug 2001). USP18 (UBP43) has been identified as a deISGylating isopeptidase (Malakhov et al. 2002). Recently, ubiquitin E2 enzymes UbcH6 and UbcH8 have been determined to function also as ISG15 conjugating enzymes (Kim et al. 2004; Zhao et al. 2004; Takeuchi et al. 2005). UbcH8 is reported to interact with several ubiquitin E3 ligases to mediate protein ubiquitylation (Dao and Zhang 2005). The fact that UbcH8 functions as a dual E2 enzyme for both ubiquitin and ISG15 raises the possibility that some UbcH8-interacting ubiquitin E3 ligases can function as an ISG15 E3 ligase. In fact, we have recently reported that the UbcH8 partner Efp ubiquitin ligase functions as

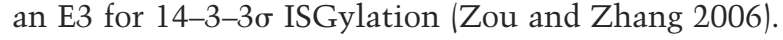

[Keywords: ISG15 (UCRP); 4EHP; ubiquitin; ISGylation; RNA cap structure; mRNA translation; interferon]

${ }^{1}$ Corresponding author.

E-MAIL dzhang@scripps.edu; FAX (858) 784-9593.

Article is online at http://www.genesdev.org/cgi/doi/10.1101/gad.1521607.
The binding of eukaryotic translation initiation factor $4 \mathrm{~F}$ (eIF4F) to the mRNA 5' cap structure is the rate-limiting step of cap structure-dependent translation initiation in eukaryotes (Gingras et al. 1999). eIF4F contains cap-binding protein eIF4E, scaffold protein eIF4G, and RNA helicase eIF4A. The interaction between eIF4E and eIF4G results in a conformational change of both proteins and enhances the association between eIF4E and the RNA cap structure (Gross et al. 2003). There are three eIF4E-family members in mammals termed eIF4E-1 (eIF4E), eIF4E-2 (4EHP and 4E-LP), and eIF4E-3 (Rom et al. 1998; Joshi et al. 2004). Like prototypical eIF4E, 4EHP is expressed ubiquitously; however, expression of eIF4E-3 is detected only in heart, skeletal muscle, lung, and spleen (Joshi et al. 2004). Similar to eIF4E, both 4EHP and eIF4E-3 bind to the RNA 5' cap structure (Rom et al. 1998; Joshi et al. 2004). Furthermore, both eIF4E and eIF4E-3 are able to bind to eIF4G to facilitate translation initiation (Rom et al. 1998; Joshi et al. 2004). However, 4EHP does not interact with eIF4G (Rom et al. 1998; Joshi et al. 2004) and thus cannot function in ribosome recruitment. This suggests that 4EHP competes with eIF4E or eIF4E-3 for binding to the RNA 5' cap structure and prevents translation. In fact, the Drosophila 4EHP homolog (d4EHP) has recently been reported to be an mRNA-specific translation inhibitor (Cho et al. 2005).

Here, we report that 4EHP is a target of ISG15 modification. The UbcH8-interacting E3 ligase HHARI (human homolog of Drosophila ariadne) (Moynihan et al. 1999; Tan et al. 2003) promotes ISGylation of 4EHP. More importantly, ISG15 modification of 4EHP substantially enhances its cap structure-binding activity. This is the first report that shows "gain of function" due to ISG15 modification and suggests a role for protein ISGylation in cap structure-dependent translation control in innate immune responses.

\section{Results and Discussion}

\section{EHP is an ISG15 target protein}

Given that $\mathrm{UbcH} 8$ is able to act as a conjugating enzyme in both the ubiquitylation and ISGylation pathways, we studied whether the UbcH8-interacting RING-IBRRING-type ubiquitin ligase HHARI can function as an ISG15 ligase to modify its target protein 4EHP (Moynihan et al. 1999; Tan et al. 2003). 4EHP could be modified by ISG15 with or without exogenous expression of HHARI when 4EHP was overexpressed in the presence of ISG15 E1 and E2. We observed two ISGylated Flag-4EHP bands (Fig. 1A). ISGylation of 4EHP was slightly enhanced by HHARI expression (Fig. 1A,B). However, HHARI $(\Delta \mathrm{C})$ that lost the UbcH8-interacting RING-IBRRING domain (Moynihan et al. 1999; Tan et al. 2003) did not increase the ISGylation of 4EHP (Fig. 1B). We further examined the ISGylation of endogenous 4EHP (Fig. 1C). ISGylation of endogenous 4EHP was clearly detected with the expression of HA-HHARI. These data showed that HHARI is an ISG15 ligase for 4EHP. Endogenous 4EHP also gave two bands of ISGylation signal, even though the top band overlapped with a nonspecific band (Fig. 1C). We also studied the ISGylation of endogenous $4 \mathrm{EHP}$ in response to IFN stimulation using UBP43 


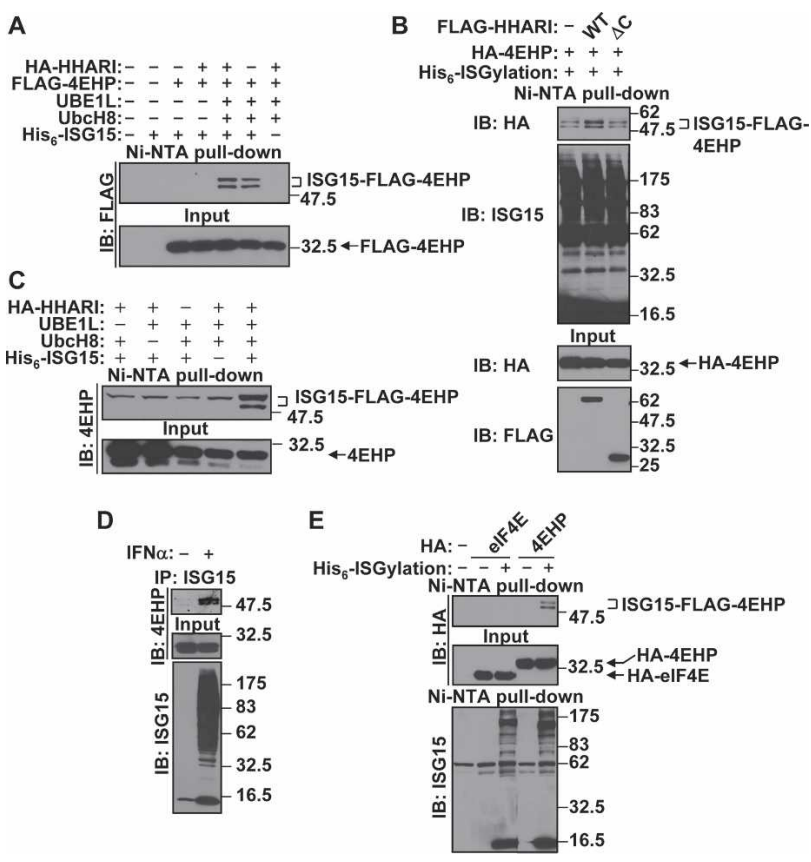

Figure 1. 4EHP is an ISGylation target. (A) ISGylation of 4EHP 293T cells were transfected with HA-HHARI, Flag-4EHP, UBE1L, $\mathrm{UbcH8}$, and $\mathrm{His}_{6}$-ISG15 expression plasmids as indicated. Thirty-six hours after transfection, cell lysates were subjected to Ni-NTA pulldown and immunoblot (IB) analysis with anti-Flag antibody. The positions of unmodified and ISGylated 4EHP are shown on the right side as well as the molecular weight markers (in kilodaltons). $(B)$ HHARI enhances ISGylation of 4EHP. Either Flag-HHARI (WT) or C-terminal-deleted Flag-HHARI $(\Delta \mathrm{C})$ was transfected to $293 \mathrm{~T}$ cells with HA-4EHP and the His ${ }_{6}$-ISGylation system (UBE1L, UbcH8, and $\mathrm{His}_{6}$-ISG15). Cell lysates were subjected to Ni-NTA pull-down and immunoblot (IB) analysis. (C) ISGylation of endogenous 4EHP. $293 \mathrm{~T}$ cells were transfected with UBE1L, UbcH8, HA-HHARI, and His $_{6}$-ISG15 expression plasmids as indicated. Cell lysates were subjected to Ni-NTA pull-down and immunoblot (IB) analysis. $(D)$ ISGylation of endogenous 4EHP dependent on IFN $\alpha$ treatment. KT-1dUBP43 cells were stimulated by $1000 \mathrm{U} / \mathrm{mL}$ of IFN $\alpha-2$ a for 48 h. The cell lysates were subjected to immunoprecipitation (IP) with anti-ISG15 antibody and immunoblot (IB) analysis. (E) eIF4E is not ISGylated. $293 \mathrm{~T}$ cells were transfected with HA-eIF4E or HA-4EHP with or without the His ${ }_{6}$-ISGylation system as indicated. Cell lysates were subjected to Ni-NTA pull-down and immunoblot (IB) analysis. The positions of unmodified eIF4E and 4EHP are shown as well as ISGylated 4EHP on the right side.

knockdown KT-1/A3 cells, which give more ISGylated protein than parental cells (Fig. 1D). Two bands of ISGylated 4EHP protein were also detected on a Western blot. Together, these results indicate that 4EHP is an ISG15 target and HHARI functions as an ISG15 E3 ligase for 4EHP modification in the presence of the ISGylation system.

Since 4EHP is $30 \%$ identical and $60 \%$ similar to eIF4E at the amino acid level (Rom et al. 1998), we also examined whether eIF4E could be modified by ISG15. HAeIF4E or HA-4EHP was expressed in 293T cells in the presence or the absence of ISGylation components. The ISGylation of 4EHP was clearly observed; however, eIF4E was not modified by ISG15 (Fig. 1E).

\section{Lys 134 and Lys 222 of 4EHP are target sites} of ISGylation

To identify the ISGylated lysines of 4EHP, $\mathrm{His}_{6}$-ISG15modified Flag-4EHP was purified by two-step purifica-

tion using Ni-NTA agarose and anti-Flag M2 agarose (Fig. 2A). Bands A and B were subjected to mass spectrometry analysis. As shown in Figure $2 \mathrm{~B}$, the peptides from band A are underlined (\#A1-A6), and those from band $\mathrm{B}$ are boxed (\#B1-B4). If any lysine residue of 4EHP was modified by ISG15, the modified lysine residue could not be recognized by trypsin and the unique peptide that contains an additional Gly-Gly from ISG15 might be detected by nano LC-MS/MS analysis (Zou et al. 2005). The mass of one peptide (\#B3) from band B matched the Gly-Gly modification (Fig. 2B). However, we could not detect the peptide that was modified by ISG15 in band A. To confirm that the candidate Lys 222 is one of the ISGylation sites, we mutated this Lys to Arg, and then examined whether this mutant 4EHP(K222R) was modified by ISG15 (Fig. 2C). Mutant 4EHP(K222R) lost the bottom ISGylated band and still kept the top ISGylated band. This result demonstrates

A
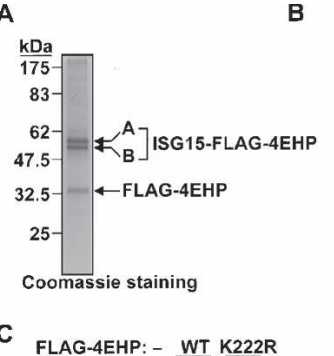

C FLAG-4EHP: - WT K222R
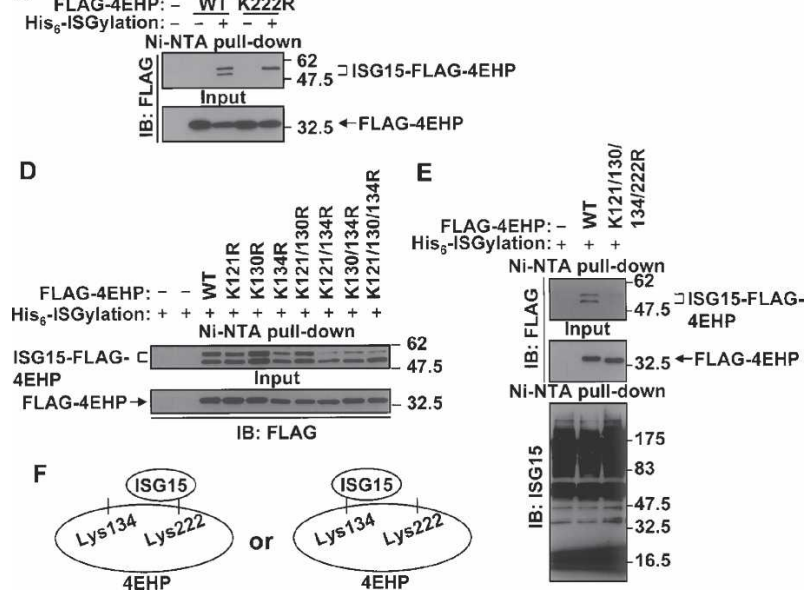

Figure 2. The ISGylation sites of 4EHP. (A) Coomassie staining of purified Flag-4EHP and ISGylated Flag-4EHP. Both bands A and B, which are ISGylated Flag-4EHP, were subjected to nano LC-MS/MS analysis. $(B)$ Identified peptides by nano LC-MS/MS analysis. Peptides that came from band A are underlined (\#A1-A6), and those that came from band B are boxed (\#B1-B4). The lysine residue that was identified to be modified by Gly-Gly is indicated by the arrow. $(C)$ K222R mutation eliminates the ISGylated bottom band of 4EHP. Either wild-type or K222R mutant Flag-4EHP was transfected to

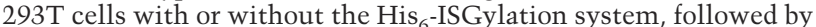
Ni-NTA pull-down and immunoblotting (IB) analysis. (D) ISGylation of Lys 134 contributes to the ISGylated top band of 4EHP. Flag-4EHP (wild type or mutants) were transfected to 293T cells with the His $_{6}$-ISGylation system as indicated, followed by Ni-NTA pull-down and immunoblotting (IB) analysis. (E) K121/130/134/ 222R mutant 4EHP is not ISGylated. Flag-4EHP (wild type or K121/ $130 / 134 / 222 \mathrm{R}$ ) was transfected to $293 \mathrm{~T}$ cells with the $\mathrm{His}_{6}$-ISGylation system as indicated, followed by Ni-NTA pull-down and immunoblotting (IB) analysis. (F) Schematic model of the ISGylation of 4EHP. Lys 134 or Lys 222 of 4EHP can be ISGylated. The ISGylation of one of the lysine residues blocks modification on another lysine residue by structural obstruction. 


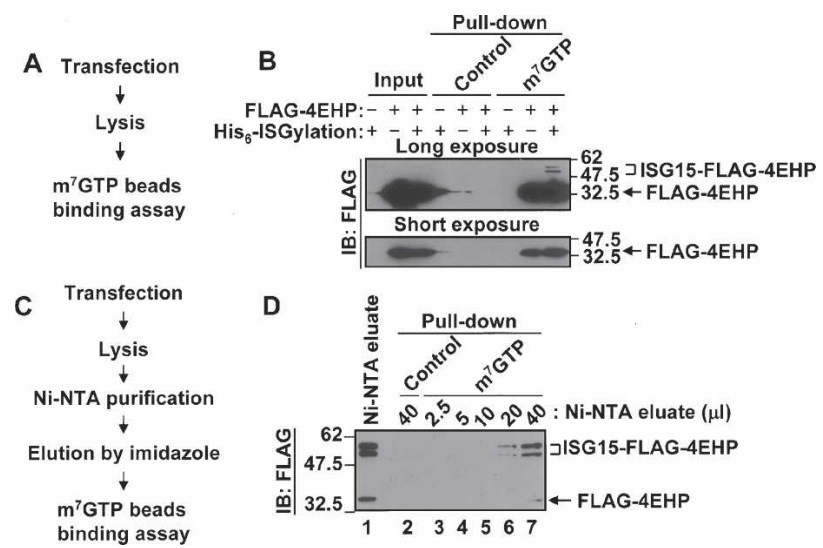

Figure 3. ISGylation of 4EHP enhances $\mathrm{m}^{7} \mathrm{GTP}$ cap structure-binding activity. $(A)$ The flow chart of the direct cap structure-binding assay. (B) Flag-4EHP was transfected to $293 \mathrm{~T}$ cells with or without His $_{6}$-ISGylation system. Cell lysates were incubated with Protein A Sepharose or $\mathrm{m}^{7}$ GTP-Sepharose, followed by immunoblotting (IB) analysis. Short and long exposures are shown. $(C)$ The flow chart of the purification of ISGylated 4EHP and nonmodified 4EHP for the cap structure-binding assay. 293T cells were transfected with Flag4EHP, HA-HHARI, and $\mathrm{His}_{6}$-ISGylation system. Cell lysates were subjected to Ni-NTA purification. The Ni-NTA-bound proteins were eluted by imidazole, and then used for the cap structure-binding assay using $\mathrm{m}^{7} \mathrm{GTP}$ beads. $(D)$ The cap structure-binding assay. After Ni-NTA purification, the eluate fraction was subjected to immunoblot (IB) analysis to show the relative amount of Flag-4EHP and ISGylated Flag-4EHP (left, lane 1). Different amounts of NiNTA eluate fraction were subjected to pull-down assay with control beads or $\mathrm{m}^{7}$ GTP beads as indicated (lanes 2-7). The positions of unmodified and ISGylated 4EHP are shown on the right side.

that 4EHP has at least two ISGylation sites, one of which is Lys 222. The ISGylation of different Lys residues results in different migration rates in SDS-PAGE. Based on the structure of eIF4E (Marcotrigiano et al. 1997) and the above mass spectrometry data (Fig. 2B), we examined Lys 121, Lys 130, and Lys 134 of 4EHP for ISGylation. These Lys residues were mutated to Arg and used for an ISGylation assay (Fig. 2D). The 4EHP(K134R) mutant partially lost the top ISGylated band, and additional mutations (K121R, K130R, or both) further decreased the top band. These data suggest that the major ISGylation site, which contributes to the top ISGylated band, is Lys 134, but Lys 121 and Lys 130 could compensate for the K134R mutation. We further constructed a 4EHP (K121/130/134/ 222R) mutant, which had similar $\mathrm{m}^{7} \mathrm{GTP}$ cap structurebinding activity (data not shown). However, this mutant almost completely lost both ISGylated bands (Fig. 2E). Based on these data, we concluded that the ISGylation of Lys 222 contributes to the bottom ISGylated band and that of Lys 134 contributes to the top ISGylated band. The ISGylation of each of these Lys residues may inhibit the modification of another Lys due to structural obstructions.

\section{ISGylation of 4EHP enhances its $\mathrm{m}^{7} \mathrm{GTP}$ cap structure-binding activity}

We next examined the cap structure-binding activity of ISGylated 4EHP. We transfected Flag-4EHP with or without the ISGylation system, and then cell lysates were directly used for a cap structure-binding assay (Fig. 3A). We did not detect ISGylated 4EHP in the input lane; however, it was clearly detected after $\mathrm{m}^{7} \mathrm{GTP}$ pull-down
(Fig. 3B). This result indicates that ISGylated 4EHP has higher cap structure-binding activity. We further confirmed this finding by another experiment shown in Figure 3C. We purified comparable amounts of ISGylated 4EHP and nonmodified 4EHP after the Ni-NTA purification. Nonmodified 4EHP was pulled down by nonspecific interaction with Ni-NTA agarose. The eluate fraction was used for the cap structure-binding assay. ISGylated 4EHP had higher cap structure-binding activity compared with nonmodified 4EHP (Fig. 3D). These data indicate that ISGylation of 4EHP causes a gain of function of 4EHP. We further confirmed this result using ISG15-4EHP fusion proteins (Fig. 4A). Such ubiquitinlike fusion proteins have been found to mimic the constitutively modified state of a protein and are particularly useful when the native modification site occurs near the terminus of a protein of interest (Ross et al. 2002). ISG15, 4EHP, and 4EHP fused to ISG15 were expressed in $293 \mathrm{~T}$ cells, and the lysates were used for the cap structure-binding assay (Fig. 4B). ISG15 alone was not able to bind to the cap structure, and 4EHP could bind to the cap structure. As we expected, if ISG15 was fused to 4EHP either N-terminally or C-terminally, the cap structure-binding activity was strongly enhanced, and the C-terminal fusion protein (Flag-4EHP-ISG15)

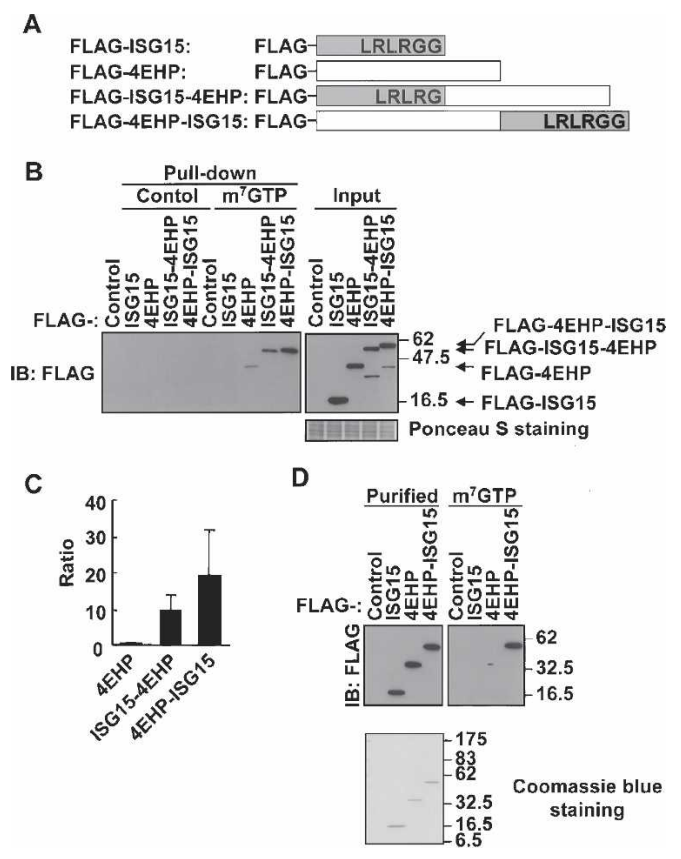

Figure 4. ISG15-4EHP fusion proteins have much higher $\mathrm{m}^{7} \mathrm{GTP}$ cap structure-binding activity. (A) Schematic representation of wildtype and ISG15-4EHP fusion proteins. $(B)$ The cap structure-binding assay using wild-type or fusion proteins. 293T cells were transfected with Flag-tagged proteins as indicated, and then equal amounts of cell lysates were subjected to the cap structure-binding assay and immunoblot (IB) analysis. Ponceau S staining shows the relative amount of protein in each sample. (C) The quantification of cap structure-binding activity of 4EHP and that fused to ISG15. The amount of protein bound to $\mathrm{m}^{7} \mathrm{GTP}$ beads, which was determined by scanning densitometry of the immunoblot, was divided by the signal intensity of the input lane and normalized by that of Flag4EHP. Data are expressed as means + standard deviations from three independent experiments. $(D)$ Cap structure-binding assay with purified proteins. Each Flag-protein was purified from 293T cells and used for cap structure-binding assay. Coomassie staining shows no interacting protein with each purified protein. 
has the highest activity. Next, we quantified the amount of Flag-4EHP or Flag-4EHP fused to ISG15 that bound to the cap structure (Fig. 4C). N- or C-terminal fusion protein has $\sim 10$ or 20 times higher cap structure-binding activity compared with Flag-4EHP itself, respectively. We also used purified proteins in the assay to verify whether other proteins in the lysates are involved in the enhanced cap structure-binding activity of 4EHP-ISG15 (Fig. 4D). Purified 4EHP-ISG15 fusion protein still had higher cap structure-binding activity compared with nonmodified 4EHP. These data strongly indicate that ISGylation of 4EHP enhances cap structure-binding activity and the ISG15-4EHP fusion protein can mimic the ISGylated 4EHP.

\section{ISGylation enhances the function of $4 E H P$ in translational control}

4EHP inhibits Caudal mRNA translation via interaction with Bicoid in Drosophila (Cho et al. 2005). Since no human 4EHP target is known yet, we investigated whether ISGylation of 4EHP could enhance the cap structure-binding activity and inhibit the translation of a specific mRNA using the Caudal-Bicoid model. We first demonstrated that both human 4EHP (Fig. 5A) and 4EHP-ISG15 (Fig. 5B) bound to Bicoid. Next, using the same assay system as described by Cho et al. (2005), we showed that human 4EHP inhibited the translation of mRNA with a Bicoid-binding site like Drosophila 4EHP in the presence of Bicoid as reported previously (Cho et al. 2005). More importantly, 4EHP-ISG15 fusion protein had higher translation inhibition activity compared with 4EHP (Fig. 5C). These data indicate that ISGylation of 4EHP enhances the cap structure-binding activity and translation-inhibition activity of 4EHP on a specific mRNA.

Although ISG15 has been known for about three decades (Farrell et al. 1979), the role of ISG15 modification remains largely unclear. Research on ISGylation has been expanded by the identification of the enzymes involved in the ISG15 conjugation process and the target proteins of modification. However, the consequence of ISGylation for most of the proteins has not been identified yet. Recently, it was reported that ISGylation of the ubiquitin-conjugating enzyme Ubc13 disrupts its ubiquitin-conjugating activity (Takeuchi and Yokosawa 2005; Zou et al. 2005). The relationship of protein ubiquitylation and ISGylation has also been explored (Liu et al. 2003; Malakhov et al. 2003; Desai et al. 2006; Kim et al. 2006). Furthermore, the positive role of protein ISGylation in antiviral responses has been reported (Yuan and Krug 2001; Lenschow et al. 2005; Okumura et al. 2006). Here we report that the ubiquitin ligase HHARI promotes the ISGylation of 4EHP and such modification increase 4EHP RNA cap structure-binding activity and translational control. The discovery that ISGylation enhances 4EHP binding to the cap structure demonstrates a novel gain-of-function modulation of protein ISG15 modification, which is highly significant considering that ISG15 generally modifies a small fraction of any target protein upon IFN stimulation or pathogen infections. Furthermore, this result extends the investigation of protein ISGylation into the regulation of protein translation.

Schizosaccharomyces pombe also has a 4EHP-like protein (eIF4E2, encoded by tif452) (Ptushkina et al. 2001). It is not essential for S. Pombe. Its expression relative to eIF4E1 is increased at higher temperature. In

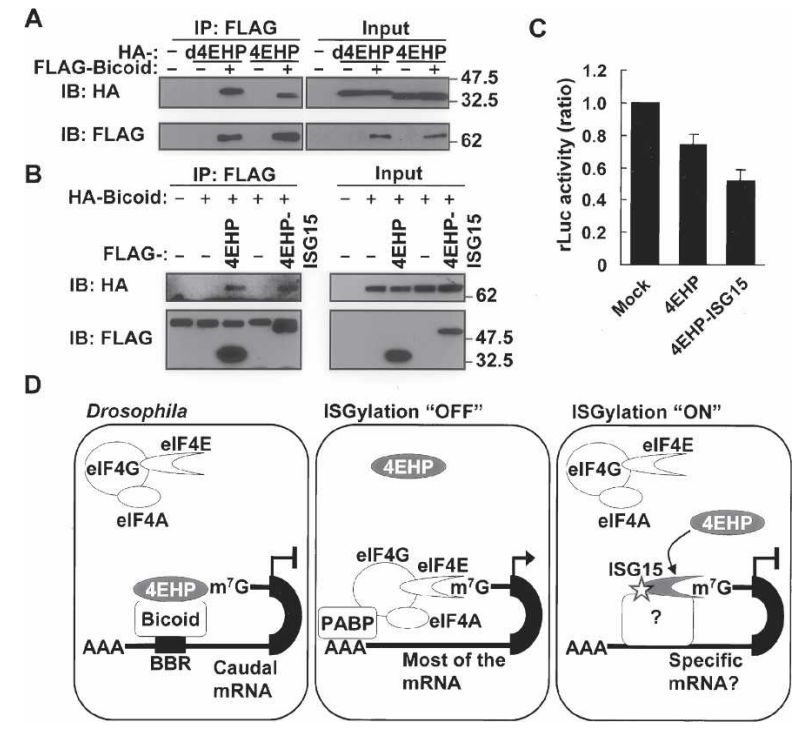

Figure 5. ISGylation of 4EHP enhances the mRNA-specific translation inhibition activity of 4EHP. (A) Human 4EHP binds to Drosophila Bicoid. HA-Drosophila 4EHP (d4EHP) or human 4EHP (4EHP) was transfected to $293 \mathrm{~T}$ cells with or without Flag-Drosophila Bicoid, followed by immunoprecipitation (IP) and immunoblot (IB) analysis. (B) Human 4EHP-ISG15 fusion protein binds to Drosophila Bicoid. Flag-tagged Human 4EHP or 4EHP-ISG15 fusion protein was transfected to $293 \mathrm{~T}$ cells with or without HA-Drosophila Bicoid, followed by immunoprecipitation (IP) and immunoblot (IB) analysis. $(C)$ In vitro translation of capped-Renilla reniformis luciferase-Bicoid-binding region reporter mRNA with purified human 4EHP or 4EHP-ISG15 fusion protein as well as mock-purified fraction. In vitro translation of the reporter mRNA was performed in the presence of in vitro transcribed and translated Drosophila Bicoid and purified human 4EHP or 4EHP-ISG15 as well as mock-purified fraction. Data are presented as mean + standard deviation from two independent experiments, both of which were duplicated $(n=4)$. The values were normalized by that obtained for mock-purified fractions. $(D)$ A model of cap structure-dependent translation regulation by ISGylated 4EHP. In Drosophila, 4EHP binds to the cap structure and Bicoid. Bicoid also binds to the Bicoidbinding region (BBR) in the $3^{\prime}$-untranslated region (UTR) of Caudal mRNA. This protein-mRNA complex prevents the interaction between eIF4E and the cap structure. As a result the translation of Caudal is inhibited. Under normal conditions (ISGylation "OFF"), the eIF4F complex-which contains eIF4A, eIF4E1, and eIF4Gtranslates mRNA in a cap structure-dependent manner in cooperation with poly(A)-binding protein (PABP). Under a particular condition (ISGylation "ON") that induces the ISGylation system, 4EHP is ISGylated, which leads to its stronger cap structure-binding activity to specific mRNA templates in cooperation with certain currently unknown factors. As a result, a lower amount of eIF4F complex binds to the cap structure of these mRNAs to initiate translation.

Caenorhabditis elegans, there are five eIF4E isoforms, termed IFE-1 through IFE-5 (Keiper et al. 2000). IFE-4 is most closely related to 4EHP and is not essential for viability (Dinkova et al. 2005). It has been reported that the translation of only some specific subsets of mRNA are affected in the ife-4 homozygous deletion mutant (Dinkova et al. 2005). Furthermore, expression of human 4EHP is increased in metastatic tumors (Ramaswamy et al. 2003). These data suggest that 4EHP is not actively involved in the main pathway of general translation initiation, but may play an active role in certain unusual conditions.

4EHP inhibits the translation of the mRNA encoding Caudal in cooperation with Bicoid in Drosophila (Fig. 5D, Drosophila; Cho et al. 2005). In normal conditions, 
eIF4E is a major regulator of translation in cooperation with other factors (Fig. 5D, ISGylation "OFF"). 4EHP has a much weaker cap structure-binding activity under the normal conditions compared with ISGylated 4EHP (Figs. $3,4)$. However, upon activation of the ISGylation system, 4EHP is modified by ISG15 and ISGylation increases its cap structure-binding activity (Fig. 5D, ISGylation "ON"). Since ISG15 itself does not have cap structure-binding activity (Fig. 4B) and another proteinprotein interaction was not required for the enhancement of cap structure-binding activity (Fig. 4D), the ISGylation of 4EHP probably causes a conformational change of 4EHP and enhances cap structure-binding activity. To test ISGylation of 4EHP in the regulation of general cap-dependent protein translation, we performed an in vitro translation assay using a capped-firefly luciferase mRNA reporter and purified 4EHP protein or 4EHP-ISG15 fusion protein. We could not detect any clear results with this assay system (data not shown). We also analyzed general translation in the presence of ISG15, 4EHP, or ISG15-4EHP fusion protein by a $\left[{ }^{35} \mathrm{~S}\right]-$ methionine metabolic incorporation experiment. No obvious difference was observed (data not shown). These results indicate that ISG15 modification of 4EHP enhances the cap structure-binding activity of 4EHP; however, it does not inhibit general translation. However, as demonstrated in Figure 5, ISGylation enhances the 4EHP inhibitory effect on translation of a specific mRNA that has a Drosophila Caudal mRNA Biocoid-binding site. Therefore, ISGylation of 4EHP may inhibit the translation of certain specific mRNAs through its interaction with other factors that are specially associated with these particular mRNAs (Fig. 5D, ISGylation "ON"). In fact, inhibition of mRNA-specific translation has also been reported in mammalian cells (Mazumder et al. 2003). For example, IFN- $\gamma$-activated inhibitor of translation (GAIT) is a $3^{\prime}$-untranslated region (UTR)-binding protein and a transcript-specific translational inhibitor. GAIT suppresses the translation of the mRNA encoding Ceruloplasmin (Cp) (Mazumder et al. 2005). Besides increasing the affinity of 4EHP for the cap structure, ISGylation may also provide a new protein interaction with factors binding to the $3^{\prime}$-UTR of certain mRNAs (Fig. 5D, ISGylation "ON"). Future studies to define 4EHP- and ISG15-interacting proteins and their target mRNA transcripts will provide valuable information about ISG15 modification in the control of specific protein translation.

It is well known that IFNs inhibit the translation of viral mRNAs while permitting normal translation of the majority of cellular mRNAs (Platanias 2005). Furthermore, IFNs induce the phosphorylation of eIF4E-binding protein (4E-BP1) to promote translation initiation (Platanias 2005). Therefore, it is possible that ISGylated 4EHP acts as a viral mRNA-specific translation inhibitor in a cap-dependent manner. Since flaviviruses, such as Dengue, West Nile, yellow fever, Kunjin, and Japanese encephalitis viruses, contain a capped positive-sense RNA genome with a nonpolyadenylated 3'-UTR (Polo et al. 1997; Edgil and Harris 2005), they may be good candidates for ISGylated 4EHP.

\section{Materials and methods}

Plasmid construction, cell culture, transfection, and antibodies Please see Supplemental Material.
Interferon stimulation

KT-1dUBP43 cells were cultured in the presence of $1000 \mathrm{U} / \mathrm{mL}$ IFNa-2a (Roche) for $48 \mathrm{~h}$ in RPMI complete medium.

Immunoprecipitation, Western blot analysis, and Ni-NTA-agarose purification

Immunoprecipitation, Western blot analysis, and Ni-NTA-agarose purification were performed as described previously (Zou and Zhang 2006) with some modifications. Please see Supplemental Material.

\section{Cap-affinity assay}

The $\mathrm{m}^{7} \mathrm{GTP}$ cap structure-affinity assay was performed as described previously (Cho et al. 2005) with some modifications. Please see Supplemental Material.

\section{Identification of the ISGylation site}

Purified $\mathrm{His}_{6}$-mISG15-conjugated Flag-4EHP was subjected to nano LCMS/MS analysis at the Scripps Research Institute mass spectrometry core facility. Please see Supplemental Material.

In vitro transcription and translation assay In vitro transcription and translation were done as described previously (Cho et al. 2005) with slight modification. Please see Supplemental Material.

\section{Acknowledgments}

We thank Dr. Nahum Sonenberg for Caudal-Bicoid translation assayrelated constructs and Dr. Ernest Borden for human ISG15 antibody. We also thank Dr. Joseph Marcotrigiano and members of the DEZ laboratory for valuable discussions and Joseph Biggs for critical editing of this manuscript. This work is supported by National Institutes of Health grants GM066955 to D.E.Z., a Uehara Memorial Foundation postdoctoral fellowship to F.O., and a Leukemia and Lymphoma Society postdoctoral fellowship to W.Z. The Stein Endowment Fund has partially supported the departmental molecular biology service laboratory for DNA sequencing and oligonucleotide synthesis. This is manuscript 18298 from The Scripps Research Institute.

\section{References}

Cho, P.F., Poulin, F., Cho-Park, Y.A., Cho-Park, I.B., Chicoine, J.D., Lasko, P., and Sonenberg, N. 2005. A new paradigm for translational control: Inhibition via $5^{\prime}-3^{\prime}$ mRNA tethering by Bicoid and the eIF4E cognate 4EHP. Cell 121: 411-423.

Dao, C.T. and Zhang, D.E. 2005. ISG15: A ubiquitin-like enigma. Front. Biosci. 10: 2701-2722.

Desai, S.D., Haas, A.L., Wood, L.M., Tsai, Y.C., Pestka, S., Rubin, E.H., Saleem, A., Nur-E-Kamal, A., and Liu, L.F. 2006. Elevated expression of ISG15 in tumor cells interferes with the ubiquitin/26S proteasome pathway. Cancer Res. 66: 921-928.

Dinkova, T.D., Keiper, B.D., Korneeva, N.L., Aamodt, E.J., and Rhoads, R.E. 2005. Translation of a small subset of Caenorhabditis elegans mRNAs is dependent on a specific eukaryotic translation initiation factor 4E isoform. Mol. Cell. Biol. 25: 100-113.

Edgil, D. and Harris, E. 2005. End-to-end communication in the modulation of translation by mammalian RNA viruses. Virus Res. 119: 43-51.

Farrell, P.J., Broeze, R.J., and Lengyel, P. 1979. Accumulation of an mRNA and protein in interferon-treated Ehrlich ascites tumour cells. Nature 279: 523-525.

Garcia-Sastre, A. and Biron, C.A. 2006. Type 1 interferons and the virushost relationship: A lesson in detente. Science 312: 879-882.

Gingras, A.C., Raught, B., and Sonenberg, N. 1999. eIF4 initiation factors: Effectors of mRNA recruitment to ribosomes and regulators of translation. Annu. Rev. Biochem. 68: 913-963.

Gross, J.D., Moerke, N.J., von der Haar, T., Lugovskoy, A.A., Sachs, A.B., McCarthy, J.E., and Wagner, G. 2003. Ribosome loading onto the mRNA cap is driven by conformational coupling between eIF4G and eIF4E. Cell 115: 739-750.

Haas, A.L., Ahrens, P., Bright, P.M., and Ankel, H. 1987. Interferon induces a 15-kilodalton protein exhibiting marked homology to ubiq- 
uitin. J. Biol. Chem. 262: 11315-11323.

Joshi, B., Cameron, A., and Jagus, R. 2004. Characterization of mammalian eIF4E-family members. Eur. J. Biochem. 271: 2189-2203.

Keiper, B.D., Lamphear, B.J., Deshpande, A.M., Jankowska-Anyszka, M., Aamodt, E.J., Blumenthal, T., and Rhoads, R.E. 2000. Functional characterization of five eIF4E isoforms in Caenorhabditis elegans. J. Biol. Chem. 275: 10590-10596.

Kim, K.I., Giannakopoulos, N.V., Virgin, H.W., and Zhang, D.E. 2004. Interferon-inducible ubiquitin $\mathrm{E} 2, \mathrm{Ubc} 8$, is a conjugating enzyme for protein ISGylation. Mol. Cell. Biol. 24: 9592-9600.

Kim, K.I., Yan, M., Malakhova, O., Luo, J.K., Shen, M.F., Zou, W., de la Torre, J.C., and Zhang, D.E. 2006. Ube1L and protein ISGylation are not essential for $\alpha / \beta$ interferon signaling. Mol. Cell. Biol. 26: $472-$ 479.

Lenschow, D.J., Giannakopoulos, N.V., Gunn, L.J., Johnston, C., O'Guin, A.K., Schmidt, R.E., Levine, B., and Virgin, H.W. 2005. Identification of interferon-stimulated gene 15 as an antiviral molecule during Sindbis virus infection in vivo. I. Virol. 79: 13974-13983.

Liu, M., Li, X.L., and Hassel, B.A. 2003. Proteasomes modulate conjugation to the ubiquitin-like protein, ISG15. J. Biol. Chem. 278: 15941602.

Loeb, K.R. and Haas, A.L. 1992. The interferon-inducible 15-kDa ubiquitin homolog conjugates to intracellular proteins. J. Biol. Chem. 267: 7806-7813

Malakhov, M.P., Malakhova, O.A., Kim, K.I., Ritchie, K.J., and Zhang, D.E. 2002. UBP43 (USP18) specifically removes ISG15 from conjugated proteins. J. Biol. Chem. 277: 9976-9981.

Malakhov, M.P., Kim, K.I., Malakhova, O.A., Jacobs, B.S., Borden, E.C., and Zhang, D.E. 2003. High-throughput immunoblotting. Ubiquitinlike protein ISG15 modifies key regulators of signal transduction. $J$. Biol. Chem. 278: 16608-16613.

Marcotrigiano, J., Gingras, A.C., Sonenberg, N., and Burley, S.K. 1997. Cocrystal structure of the messenger RNA $5^{\prime}$ cap-binding protein (eIF4E) bound to 7-methyl-GDP. Cell 89: 951-961.

Mazumder, B., Seshadri, V., and Fox, P.L. 2003. Translational control by the 3'-UTR: The ends specify the means. Trends Biochem. Sci. 28: 91-98.

Mazumder, B., Sampath, P., and Fox, P.L. 2005. Regulation of macrophage ceruloplasmin gene expression: One paradigm of 3'-UTR-mediated translational control. Mol. Cells 20: 167-172.

Moynihan, T.P., Ardley, H.C., Nuber, U., Rose, S.A., Jones, P.F. Markham, A.F., Scheffner, M., and Robinson, P.A. 1999. The ubiquitin-conjugating enzymes $\mathrm{UbcH} 7$ and UbcH8 interact with RING finger/IBR motif-containing domains of HHARI and H7-AP1. J. Biol. Chem. 274: 30963-30968.

Okumura, A., Lu, G., Pitha-Rowe, I., and Pitha, P.M. 2006. Innate antiviral response targets HIV-1 release by the induction of ubiquitin-like protein ISG15. Proc. Nat1. Acad. Sci. 103: 1440-1445.

Platanias, L.C. 2005. Mechanisms of type-I- and type-II-interferon-mediated signalling. Nat. Rev. Immunol. 5: 375-386.

Polo, S., Ketner, G., Levis, R., and Falgout, B. 1997. Infectious RNA transcripts from full-length dengue virus type 2 cDNA clones made in yeast. J. Virol. 71: 5366-5374.

Ptushkina, M., Berthelot, K., von der Haar, T., Geffers, L., Warwicker, J., and McCarthy, J.E. 2001. A second eIF4E protein in Schizosaccharomyces pombe has distinct eIF4G-binding properties. Nucleic Acids Res. 29: 4561-4569.

Ramaswamy, S., Ross, K.N., Lander, E.S., and Golub, T.R. 2003. A molecular signature of metastasis in primary solid tumors. Nat. Genet. 33: 49-54.

Rom, E., Kim, H.C., Gingras, A.C., Marcotrigiano, J., Favre, D., Olsen, H., Burley, S.K., and Sonenberg, N. 1998. Cloning and characterization of 4EHP, a novel mammalian eIF4E-related cap-binding protein. J. Biol. Chem. 273: 13104-13109.

Ross, S., Best, J.L., Zon, L.I., and Gill, G. 2002. SUMO-1 modification represses Sp3 transcriptional activation and modulates its subnuclear localization. Mol. Cell 10: 831-842.

Takeuchi, T. and Yokosawa, H. 2005. ISG15 modification of Ubc13 suppresses its ubiquitin-conjugating activity. Biochem. Biophys. Res. Commun. 336: 9-13.

Takeuchi, T., Iwahara, S., Saeki, Y., Sasajima, H., and Yokosawa, H. 2005. Link between the ubiquitin conjugation system and the ISG15 conjugation system: ISG15 conjugation to the UbcH6 ubiquitin E2 enzyme. J. Biochem. 138: 711-719.

Tan, N.G., Ardley, H.C., Scott, G.B., Rose, S.A., Markham, A.F., and Robinson, P.A. 2003. Human homologue of ariadne promotes the ubiquitylation of translation initiation factor 4E homologous protein, 4EHP. FEBS Lett. 554: 501-504.

Yuan, W. and Krug, R.M. 2001. Influenza B virus NS1 protein inhibits conjugation of the interferon (IFN)-induced ubiquitin-like ISG15 protein. EMBO I. 20: 362-371.

Zhao, C., Beaudenon, S.L., Kelley, M.L., Waddell, M.B., Yuan, W., Schulman, B.A., Huibregtse, J.M., and Krug, R.M. 2004. The UbcH8 ubiq uitin E2 enzyme is also the E2 enzyme for ISG15, an IFN- $\alpha / \beta$-induced ubiquitin-like protein. Proc. Natl. Acad. Sci. 101: 7578-7582.

Zou, W. and Zhang, D.E. 2006. The interferon-inducible ubiquitin-protein isopeptide ligase (E3) EFP also functions as an ISG15 E3 ligase. I Biol. Chem. 281: 3989-3994.

Zou, W., Papov, V., Malakhova, O., Kim, K.I., Dao, C., Li, J., and Zhang, D.E. 2005. ISG15 modification of ubiquitin E2 Ubc13 disrupts its ability to form thioester bond with ubiquitin. Biochem. Biophys. Res. Commun. 336: 61-68. 


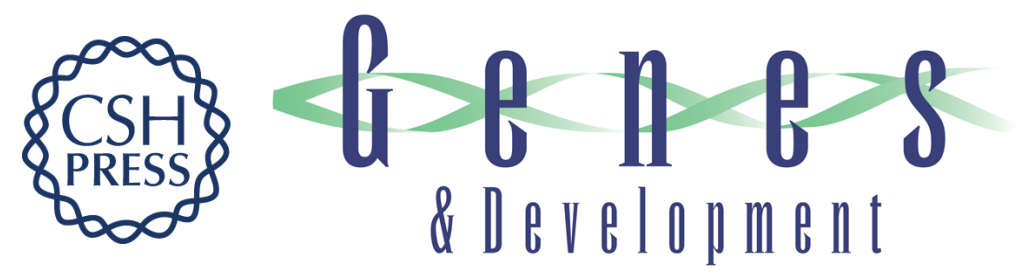

\section{ISG15 modification of the elF4E cognate 4EHP enhances cap structure-binding activity of 4EHP}

Fumihiko Okumura, Weiguo Zou and Dong-Er Zhang

Genes Dev. 2007, 21:

Access the most recent version at doi:10.1101/gad.1521607

Supplemental http://genesdev.cshlp.org/content/suppl/2007/02/08/21.3.255.DC1
Material

References This article cites 36 articles, 19 of which can be accessed free at: http://genesdev.cshlp.org/content/21/3/255.full.html\#ref-list-1

License

Email Alerting Receive free email alerts when new articles cite this article - sign up in the box at the top Service right corner of the article or click here.

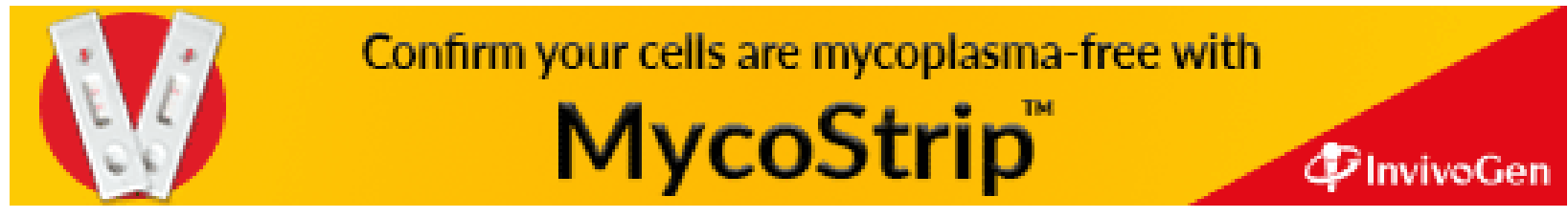

